\title{
EXPERIMENTAL STUDY OF PRESSURE DISTRIBUTIONS DUE TO THE BREAKING WAVE IMPACTS
}

\author{
Dogan Kisacik $^{1}$, Peter Troch ${ }^{1}$ and Philippe Van Bogaert
}

\begin{abstract}
Results from wave impact tests are used to analyze the pressure distribution on a vertical structure with an overhanging horizontal cantilever slab. The structure faces two individual impacts, occurring sequentially on the vertical and horizontal parts. An expression is being proposed for the location of maximum pressures $p_{\max }\left(z_{\max }\right)$ on the vertical part as a function of the wave steepness $\left(H / L_{0}\right)$. The boundary conditions for occurring of high dynamic pressures are determined both on the vertical and horizontal part. Three different pressure distribution cases are proposed as SBW, BW and BWSAT according to the breaker type. For each breaker type, the local pressure ratio's $p_{h 3} / p_{h 1}, p_{h 1} / p_{v 1}$, and $p_{h 2} / p_{v 1}$ are analyzed, where $p_{h 1}, p_{h 2}$ and $p_{h 3}$ are the local peak pressure at the SWL and top and bottom of the vertical part respectively, whereas, $p_{v 1}$ is the local peak pressure on the horizontal part. Recommended profiles are calculated from local $p_{\max }$ values.
\end{abstract}

Keywords: Vertical walls; breaking wave impact; impact pressure and force

\section{INTRODUCTION}

The Pier of Blankenberge which is located along the Belgian coast is a coastal structure consisting of a vertical core attached to an overhanging horizontal cantilever slab. Throughout high tides and storms, the structure is exposed to violent wave impacts, including waves running up against the vertical core and slamming on the horizontal deck. This introduces an important uplifting force. The lift forces consist of impact loads of high magnitude and short duration. It is reasonably impossible to substitute these impact effects by a static equivalent.

The pressure distribution due to the violent water wave impacts on a vertical wall with an overhanging horizontal cantilever slab is an important issue which is based on the kinematics of breaking waves. For the analysis, a data set from a small scale test set-up with a scale factor of 1:20 is used (see fig.1). Tests are conducted under regular waves for different values of water depth and wave period. Pressures are measured with 10 pressure sensors at various locations. The model is located 22.5 $\mathrm{m}$ away from the wave paddle on a uniform slope. The foreshore slope is $1 / 20$. The physical model is instrumented with 10 pressure sensors to register wave impact pressures and related forces both on the vertical and horizontal parts. Free surface profile and breaker shape of the waves on the vertical wall are measured from the high speed camera (HSC) recordings. Nine wave gauges are installed near the structure respectively for active wave absorption, wave reflection and breaking wave height.

The scaled model faces two individual impacts, occurring sequentially on the vertical and horizontal parts. The locations of maximum pressures $\left(p_{\max }\right)$ both on the vertical and horizontal parts are important for designing such structures. An expression is being proposed for the location of $p_{\max }$ $\left(z_{\max }\right)$ on the vertical part as a function of the wave steepness $\left(H / L_{0}\right)$. The location of $p_{\max }$ is gradually decreasing from a point above the still water level (SWL) to a point below the SWL with increasing $h_{s}$, where $h_{s}$ is the water depth at the model toe. On the horizontal part, $p_{\max }$ is located at the attached corner of the scaled model and its magnitude decreases sharply below $10 \rho g H$ between $x / h_{s}=0.8-1$.

The largest impacts recorded on the vertical part have rise times $t_{r} \leq 0.3 \mathrm{~ms}$. Similar to the pressures measured on the vertical part, an inverse relation between $p_{\max }$ and $t_{r}$ is found with a minimum rise time $t_{r} \leq 0.15 \mathrm{~ms}$ on the horizontal part. Recently, Kisacik et al. (2012) described the loading conditions due to violent wave impacts on a vertical structure with an overhanging horizontal cantilevering slab based on the data, measured at a single water depth.

The boundary conditions for occurring of high dynamic pressures are determined both on the vertical and horizontal part. Accordingly, four different pressure distribution cases are proposed as slightly breaking waves (SBW), breaking waves with small air trap (BWSAT), breaking waves with large air trap (BWLAT), and broken waves (BW) for according to the breaker type.

One should keep in mind that a phase differences exists between these local peaks. Kisacik et al. (2012) measured phase differences around $0.0272 \mathrm{~T}$ and $0.0150 \mathrm{~T}$ ( $\mathrm{T}$ is the wave period) between $p_{h 1}$ and $p_{v 1}$ for cases of SBW and breaking waves. The pressure profile at the instant of maximum horizontal or vertical force or maximum overturning moment will be different and lower than the values calculated from these profiles. Therefore, the total forces calculated from these profiles will be a

\footnotetext{
${ }^{1}$ Department of Civil Engineering, Ghent University, Technologiepark 904, Ghent, 9052, Belgium
} 
good estimation for the upper limits of forces measured on the vertical structures with an overhanging horizontal cantilever slab.

Within this paper, an overview of the small scale model test set up will be provided. This will be followed by the definition of the location of the maximum pressure. Then, the relationship between maximum pressure and rise time will be being discussed. In each breaker type the local pressure ratios are defined. Based on the discussion of the test results, conclusions will be formulated.

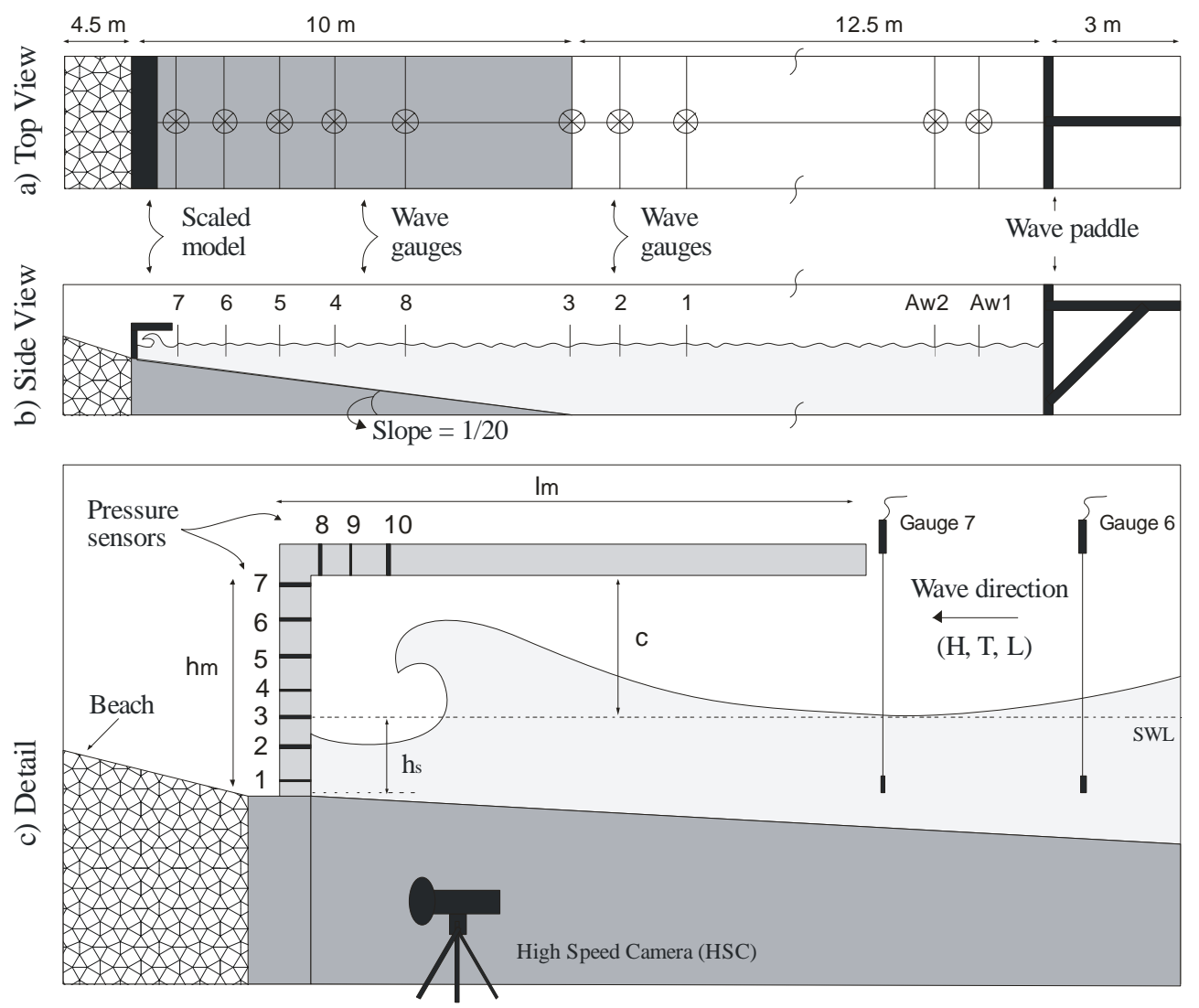

Figure 1. Small-scale model set up. a) is the top view, b) is the side view and c) is detailed view of model

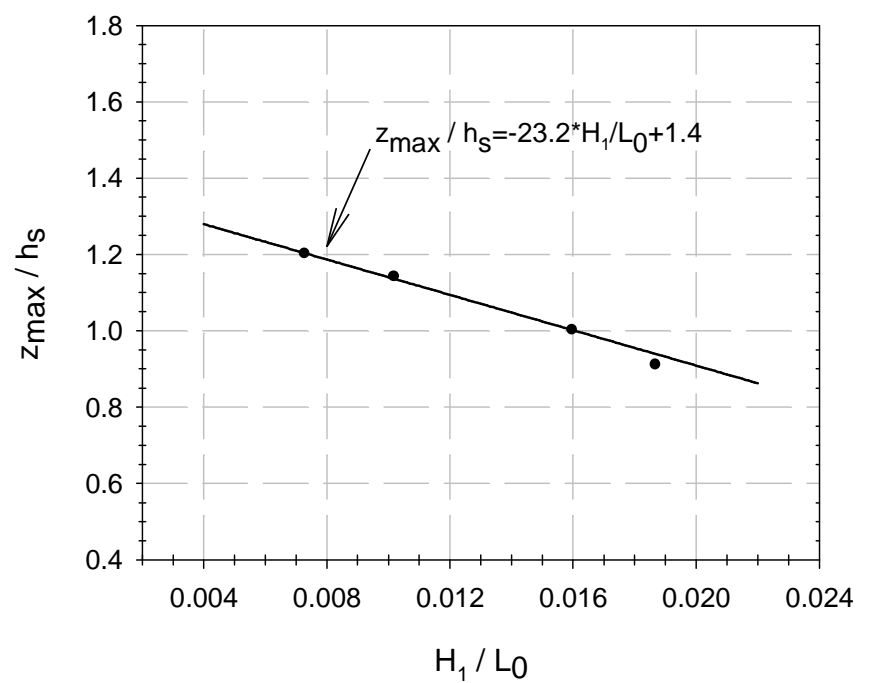

Figure 2. Variation of non-dimensional $p_{\max }$ location $\left(\mathrm{z}_{\max } / \mathrm{h}_{\mathrm{s}}\right)$ with the variation of $\mathrm{H}_{1} / \mathrm{L}_{0}$. 
LOCATION OF MAXIMUM PRESSURE, $\boldsymbol{p}_{\max }$

On the vertical structures, defining the value of $p_{\max }$ and its location $\left(z_{\max }\right)$ are quite important parameters for structural designers. In general, SWL is suggested as a good choice of $p_{\max }$ in design. Most research locates $p_{\max }$ in the vicinity of the SWL, depending on parameters like beach conditions, water depth [Kirkgoz (1982) and Hattori et al. (1994)] and breaker type [Partenscky (1988) and Hull \& Müller (2002)]. Kisacik et al. (2012) showed that $p_{\max }$ shifts from a position above SWL for SBW, to slightly below SWL for BWLAT. Similar findings are observed by Hull \& Müller (2002).

In the present work, the variation of $z_{\max }$ with $h_{s}$ is studied. For each value of $h_{s}$, a large data set which covers all breaker types from SBW to BW is considered and the highest pressures are mainly observed in the case of BWSAT.

Figure 2 shows the variation of $z_{\max } / h_{s}$ with the wave steepness $\left(H_{1} / L_{0}\right)$, where $L_{0}$ is the deep water wave length. The $H_{1}$ value is the wave height (measured at the toe of the foreshore) which results in the highest impact pressure $\left(p_{\max }\right)$ on the vertical part. The resulting expression is given in Equ. 1.

$$
z_{\max } / h_{s}=-23.2 H_{1} / L_{0}+1.4
$$

\section{PRESSURE DISTRIBUTIONS}

Figure 3 shows an example of the spatial distribution of $p_{\max }$ both on the vertical and horizontal part for cases SBW, BWSAT, BWLAT and BW. The value of $p_{h 1}, p_{h 2}$ and $p_{h 3}$ are the local peak pressure at the SWL and top and bottom of the vertical part respectively. Whereas, $p_{v 1}$ shows the local peak pressure on the horizontal part. This is the location of sensor 8 which is very close to the attached corner (see Figure 1). A combination of the maximum local peak pressures of all 10 sensors constitutes the pressure profile on the scaled model. One should keep in mind that a phase differences exist between these local peaks. For example, Kisacik et al. (2012) measured phase differences around $0.0272 \mathrm{~T}$ and $0.0150 \mathrm{~T}$ between $p_{h 1}$ and $p_{v 1}$ for cases of SBW and breaking waves (include both BWSAT and BWLAT) respectively. Therefore, pressure profile at the instant of maximum horizontal or vertical force or maximum overturning moment will be different and lower than the values calculated from these profiles. However, the total forces calculated from these profiles will be a good assessment for structural equilibrium.
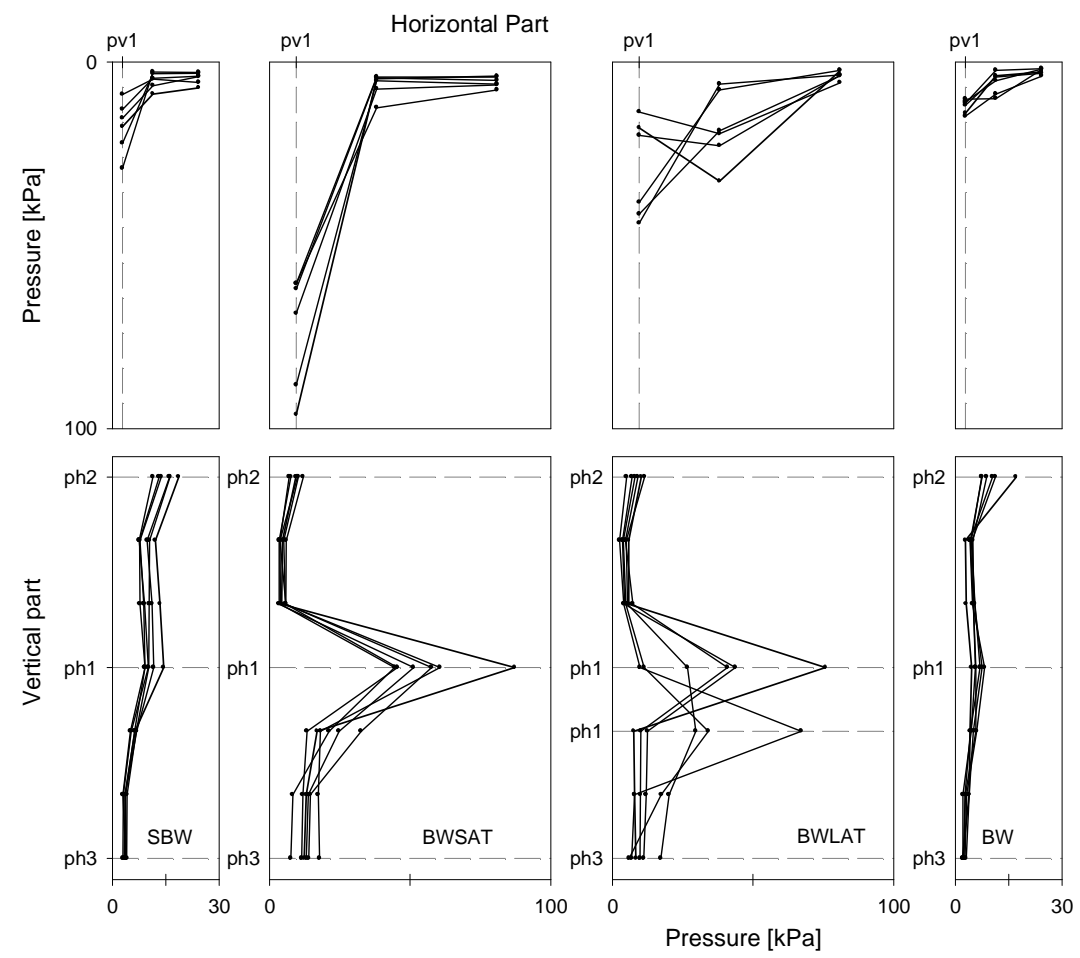

Figure 3. Spatial distribution of maximum local peak pressures $\left(p_{\max }\right)$ both on the vertical and horizontal part for cases SBW, BWSAT, BWLAT and BW $\left(h_{s}=0.105 \mathrm{~m}\right) . p_{h 1}, p_{h 2}$ and $p_{h 3}$ are the local $p_{\max }$ at various location on vertical part and $p_{v 1}$ is the local $p_{\max }$ on the horizontal part. 


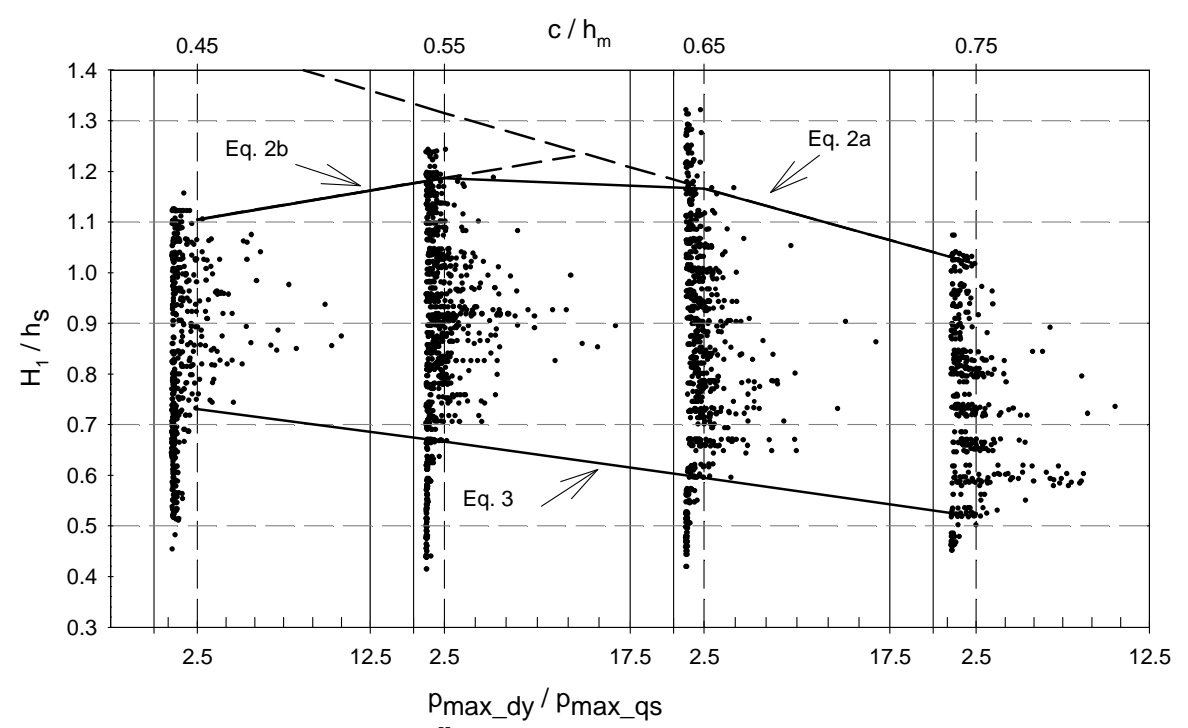

Figure 4. Variation of wave height range $\left(\frac{H_{1}}{h_{\mathrm{s}}}\right)$ which creates high dynamic pressures on the vertical part with the change of $\frac{c}{h_{m}}$. Continuous lines are measured upper and lower boundaries while dashed lines represent adopted upper boundaries. Upper and lower boundaries are determined between the points where $\boldsymbol{p}_{\text {max }_{-} d y} /$ $p_{\text {max_qs }} \geq 2.5$.

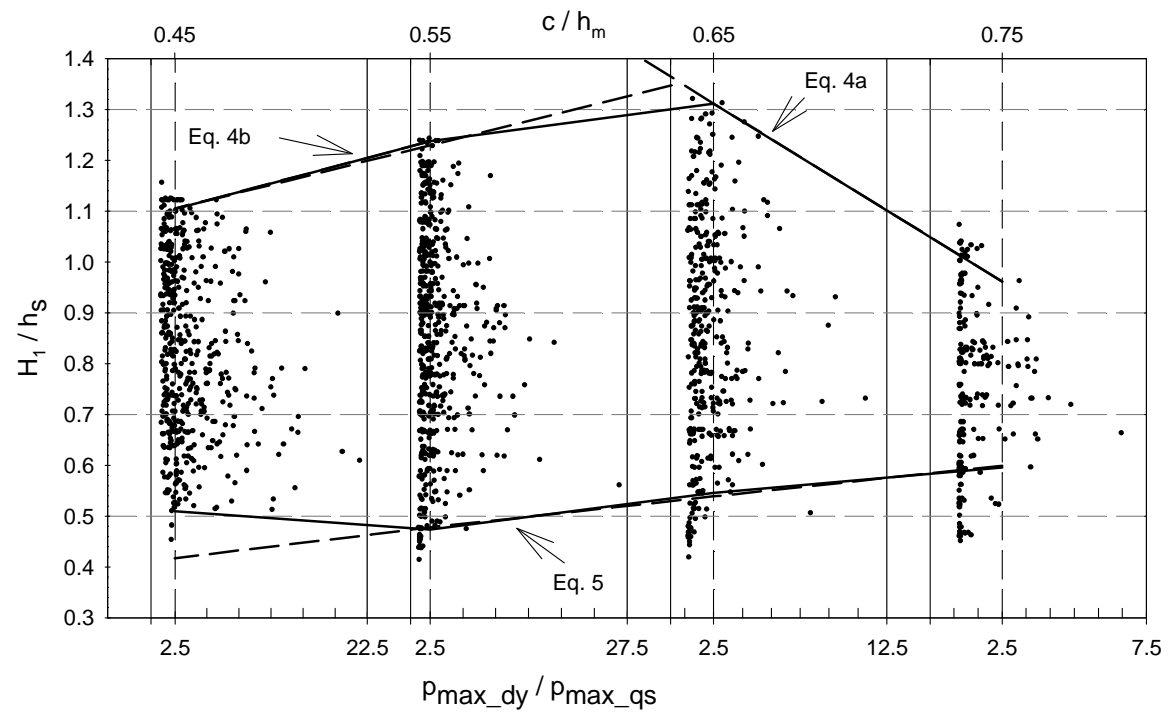

Figure 5. Variation of wave height range $\left(\frac{H}{h_{s}}\right)$ which creates high dynamic pressures on the horizontal part with the change of $\frac{c}{h_{m}}$. Continuous lines are measured upper and lower boundaries while dashed lines represent adopted boundaries. Upper and lower boundaries are determined between the points where $p_{\text {max_dy }} / p_{\text {max_qs }} \geq 2$. 5 .

\section{BOUNDARY CONDITIONS FOR OCCURRING DYNAMIC PRESSURES}

As seen from Figure 3, the pressure profiles of all four cases are quite different. Regarding design conditions, the region of occurrence of dynamic pressures is important in developing a generally feasible design method. Figure 4 shows the interval of wave height $H_{1}$ (measured at the toe of foreshore) which creates a high dynamic impact on the vertical part as a function of the clearance $(c)$ between SWL and the horizontal part. In the figure, $H_{1}$ is normalized by the water depth $h_{s}$ at the structure while $c$ is normalized by the model height $\left(h_{m}\right)$ (see Figure 1). Through the normalization, the breaking wave impact region is correlated to $h_{s}$ while $c$ is correlated to the model geometry. The bottom $x$-axis shows the ratio of the maximum dynamic and quasi-static peak pressures ( $p_{\text {max_dy }} /$ $p_{\text {max_qs }}$ ). For each $\frac{c}{h_{m}}$ value, the upper and lower boundaries of $\frac{H_{1}}{h_{s}}$ are defined at a threshold value 
corresponding to $p_{\text {max_dy }} / p_{\text {max_qs }} \geq 2.5$. This criterion for defining a dynamic pressure region is suggested by Kortenhaus and Oumeraci (1998). The continuous lines represent the measured lower and upper boundary regions. The dashed line shows the adopted upper boundary margins. Equation 2 and Equation 3 express the functions of upper and lower boundaries. 2.5:

The boundary region of the dynamic pressures exists on the vertical part where $p_{\max \_d y} / p_{\max \_q s} \geq$

Equation 2 and Equation 3 express the upper and lower boundaries, respectively.

a) for $0.60 \leq \frac{c}{h_{m}} \leq 0.75 \quad \frac{H_{1}}{h_{s}}=-1.5 \frac{c}{h_{m}}+2.14$

b) for $0.45 \leq \frac{c}{h_{m}}<0.60 \quad \frac{H_{1}}{h_{s}}=0.82 \frac{c}{h_{m}}+0.73$

for $0.45 \leq \frac{c}{h_{m}} \leq 0.75 \quad \frac{H_{1}}{h_{s}}=-0.71 \frac{c}{h_{m}}+1.05$

The lower and upper boundaries for $\frac{H_{1}}{h_{s}}$ linearly decrease with the increase of $\frac{c}{h_{m}}$ in the zone of $0.60 \leq \frac{c}{h_{m}} \leq 0.75$. In this zone, both Equation 2a and Equation 3 are more or less parallel or little expanding. Consequently, the occurring region of dynamic pressure is around $0.5 \frac{H_{1}}{h_{s}}$. However, the upper boundary shows lower values in the zone of $\frac{c}{h_{m}}<0.65$. This is the area where $H_{1}$ ranges are incomplete because of the rebounding effect of the horizontal part. The reduction in the upper boundary reaches $24 \%$ at $\frac{c}{h_{m}}=0.45$. Therefore, the upper boundary is modified in the zone of $\frac{c}{h_{m}}<0.65$ (Equation 2b).

Figure 5 shows the interval of $\frac{H_{1}}{h_{s}}$, which creates a high dynamic impact on the horizontal part. Equation 4 and Equation 5 express the upper and lower boundaries for $\frac{H_{1}}{h_{s}}$, respectively. Both equations express that the area of dynamic pressures is getting larger with the decrease of clearance. As in the case of the vertical part, measured upper boundary shows lower values in the zone of $\frac{c}{h_{m}} \leq 0.64$. However, the reduction in this case is more significant than the reduction for the case of the vertical part. This can be explained by the fact that $H_{1}$ range, creating high dynamic pressures on the horizontal part, is larger than the range creating high dynamic pressures on the vertical part. Equation $4 \mathrm{~b}$ expresses the modified upper boundary in the zone of $\frac{c}{h_{m}}<0.64$.

The boundary region of the dynamic pressures exists on the horizontal part where $p_{\text {max_dy }} /$ $p_{\text {max_qs }} \geq 2.5$. Equation 4 and Equation 5 express the upper and lower boundaries, respectively.

$\begin{array}{ll}\text { a) for } 0.64 \leq \frac{\mathrm{c}}{\mathrm{h}_{\mathrm{m}}} \leq 0.75 & \frac{\mathrm{H}_{1}}{\mathrm{~h}_{\mathrm{s}}}=-3.5 * \frac{\mathrm{c}}{\mathrm{h}_{\mathrm{m}}}+3.59 \\ \text { b) for } 0.45 \leq \frac{\mathrm{c}}{\mathrm{h}_{\mathrm{m}}}<0.64 & \frac{\mathrm{H}_{1}}{\mathrm{~h}_{\mathrm{s}}}=1.33 * \frac{\mathrm{c}}{\mathrm{h}_{\mathrm{m}}}+0.51 \\ \text { for } 0.45 \leq \frac{\mathrm{c}}{\mathrm{h}_{\mathrm{m}}} \leq 0.75 & \frac{\mathrm{H}_{1}}{\mathrm{~h}_{\mathrm{s}}}=0.61 * \frac{\mathrm{c}}{\mathrm{h}_{\mathrm{m}}}+0.14\end{array}$

\section{PRESSURE PROFILES AT SBW}

In the following, the pressure profiles of four cases (SBW, BWSAT, BWLAT and BW) are discussed based on the pressure distributions shown in Figure 3. Pressure values represent the local maximum peak pressure at specified locations. The boundary conditions of all four cases are determined based on the classification according to the breaker type on the vertical part. The boundary region of $\frac{H_{1}}{h_{S}}$ for the first case (SBW) is the area below Equation 3 where $p_{\text {max } \_y} / p_{\text {max } q \text { s }} \leq 2.5$ and $0.45 \leq \frac{c}{h_{m}}<0.60$. 

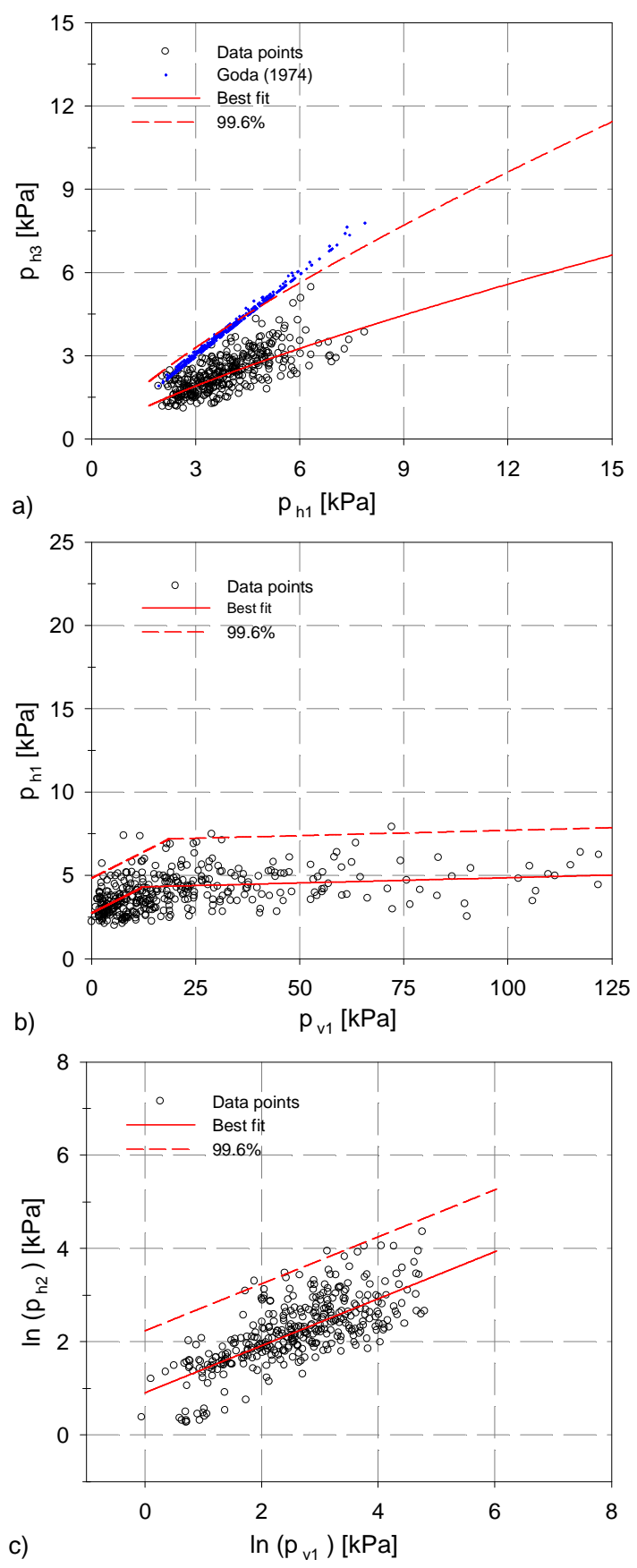

Figure 6. Evaluation of maximum pressure profiles in the case of SBW. a) $\left.\left.p_{\mathrm{h} 3} / \mathrm{p}_{\mathrm{h} 1}, \mathrm{~b}\right) \mathrm{p}_{\mathrm{h} 1} / \mathrm{p}_{\mathrm{v} 1}, \mathrm{c}\right) \mathrm{p}_{\mathrm{h} 2} / \mathrm{p}_{\mathrm{v} 1}$

Once the maximum pressures on the vertical or horizontal part $\left(p_{h 1}\right.$ or $\left.p_{v 1}\right)$ are known, the relations for $p_{h 3} / p_{h 1}, p_{h 1} / p_{v 1}$, and $p_{h 2} / p_{v 1}$ can be determined. Figure 6 a shows the relation between maximum local peak pressures at the toe of the vertical part $\left(p_{h 3}\right)$ and maximum local peak pressures at the SWL $\left(p_{h 1}\right)$. An exponential relation between $p_{h 3}$ and $p_{h 1}$ is observed. The results are compared to the theoretical expression of Goda (1974) for pulsating waves. The Goda values are calculated under the same geometric and hydrodynamic conditions. Even Goda's method slightly underestimates the small value of $p_{h 3}$. One should bear in mind that Goda's method is developed for pressure distributions at the time of maximum horizontal force on a simple vertical wall which will be slightly different. The proposed line merges with Goda line for most of the $p_{h 1}$ values. The Equation 6 represents the mean of the present data by a standard deviation $\mathrm{s}=0.2057$.

$$
\ln \left(p_{h 3}\right)=0.77 \ln \left(p_{h 1}\right)-0.2
$$



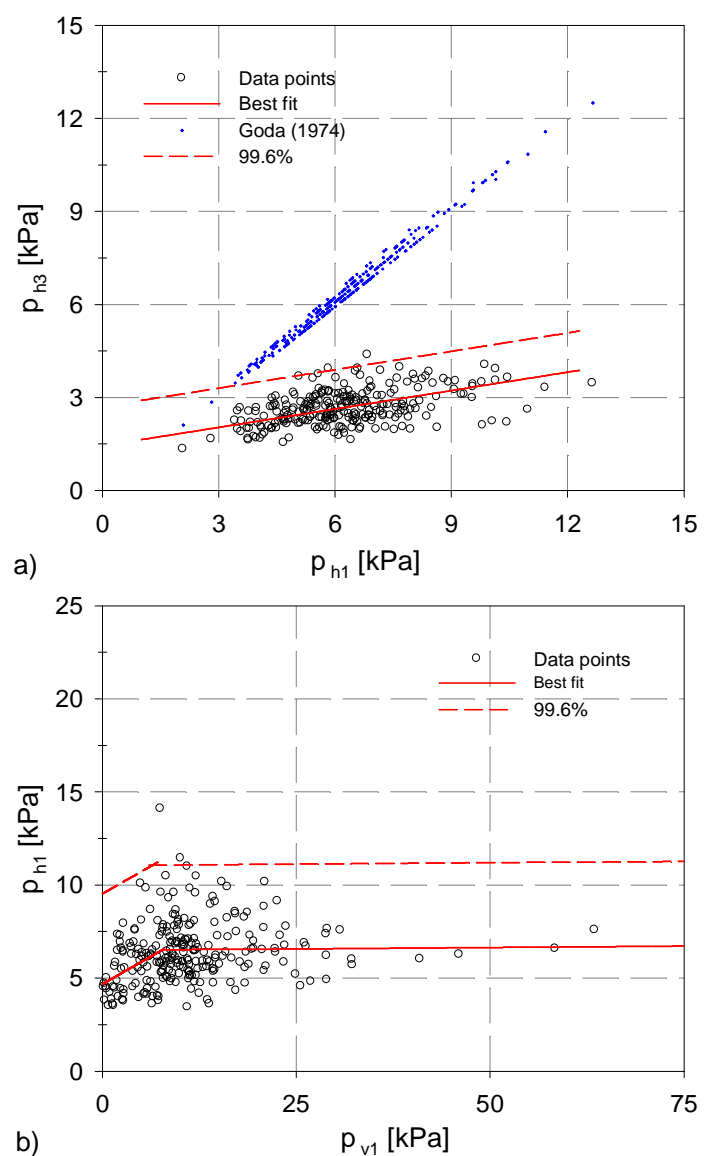

b)

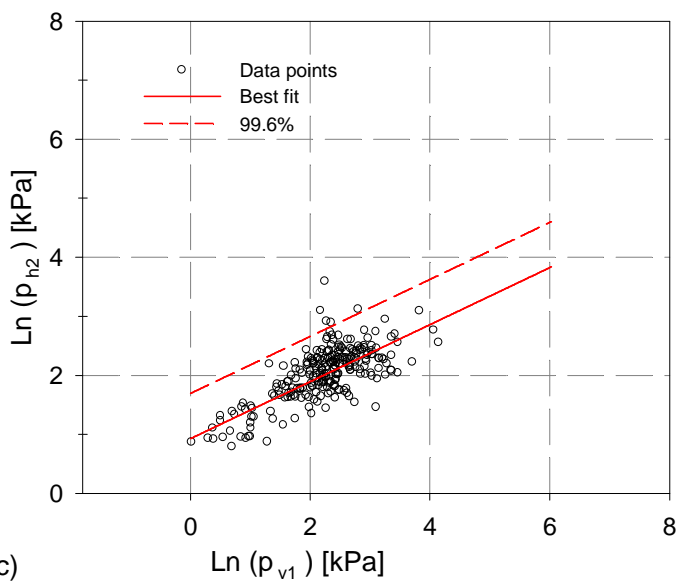

Figure 7. Evaluation of maximum pressure profiles in the case of BW. a) $p_{h 3} / p_{h 1}$, b) $p_{h 1} / p_{v 1}$, c) $p_{h 2} / p_{v 1}$

Figure $6 \mathrm{~b}$ shows the relation between $p_{h 1}$ and the maximum pressure on the horizontal part $\left(p_{v 1}\right)$. $p_{v 1}$ values are calculated from sensor 8 which is the sensor closest to the vertical part (see Figure 1). In the case of SBW, the wave tends to break but the water level at the wall accelerates fast and results in an incomplete breaking due to the presence of the wall. Therefore, most of the energy reflects from the wall and the structure is subjected to pulsating loads. The magnitude of this pulsating pressure does not exceed $8 \mathrm{kPa}$. However, the accelerated vertical component collides on the horizontal part as an uprising water jet. This water jet results in a very high impact pressure (up to $125 \mathrm{kPa}$ ). For very small values of $p_{h 1}$ and $p_{v 1}$, a linear relation is observed and this relation extends up to the maximum quasistatic pressure of $p_{h 1}$. Hence, a constant value of $p_{h 1}$ for longer values of $p_{v 1}$ may be assumed (Equation 7).

$p_{v 1} \leq 12 \quad p_{h 1}=0.13 p_{h 1}+2.75$ with $s=0.789 \quad p_{v 1}>12 \quad p_{h 1}=4.5 \quad$ with $s=1.07$ 


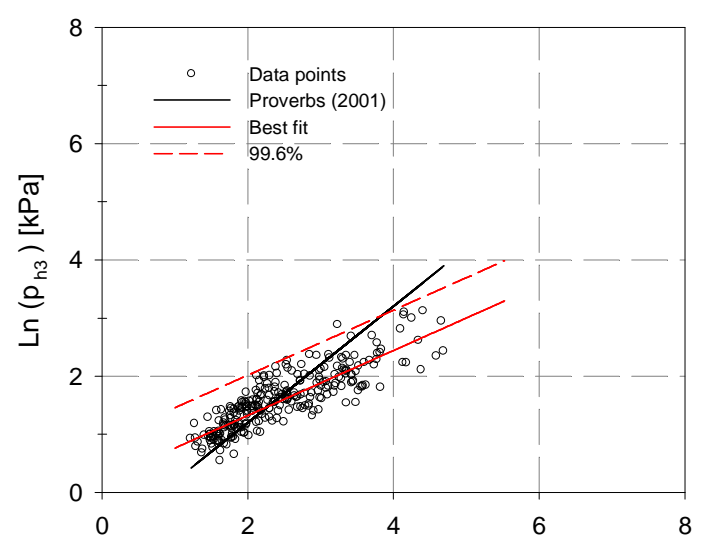

a)

$\operatorname{Ln}\left(\mathrm{p}_{\mathrm{h} 1}\right)[\mathrm{kPa}]$

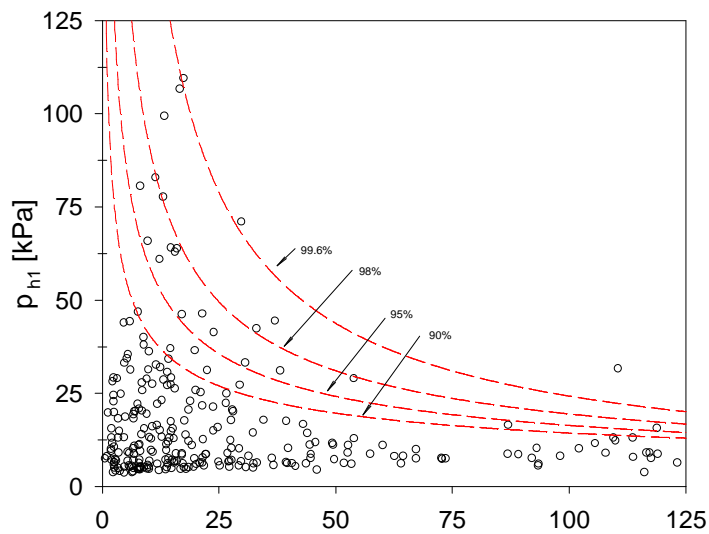

b)

$\mathrm{p}_{\mathrm{v} 1}[\mathrm{kPa}]$

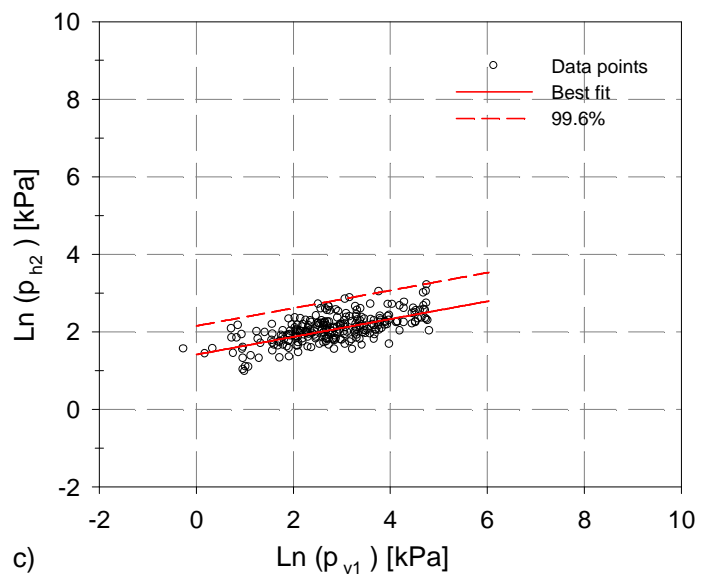

Figure 8. Evaluation of maximum pressure profiles in the cases of BWSAT. a) $p_{\mathrm{h} 3} / \mathbf{p}_{\mathrm{h} 1}$, b) $\left.\mathrm{p}_{\mathrm{h} 1} / \mathbf{p}_{\mathrm{v} 1}, \mathrm{c}\right) \mathrm{p}_{\mathrm{h} 2} / \mathbf{p}_{\mathrm{v} 1}$

Figure $6 \mathrm{c}$ expresses the relation between the maximum pressure at the upper corner of the vertical part $\left(p_{h 2}\right)$ and $p_{v 1}$. The values of $p_{h 2}$ are calculated from sensor 7 (see Figure 1). The impact force due to the water jet rising on the vertical part is the driving force for both pressures. The water jet first hits beneath the horizontal part and then influences the measurements at sensor 7 . Therefore, measured $p_{v 1}$ values are significantly higher than $p_{h 2}$ values. A logarithmic relation (Equation 8 ) is considered for the mean function with $\mathrm{s}=0.4882$. When the clearance is reduced, both $p_{h 2}$ and $p_{v 1}$ values increase.

$$
\ln \left(p_{h 2}\right)=0.51 \ln \left(p_{v 1}\right)+0.88
$$




\section{PRESSURE PROFILES AT BW}

The boundary region of $\frac{H}{h_{\mathrm{s}}}$ for $\mathrm{BW}$ is the area above Equation 2 . In this region $p_{\text {max_dy }} / p_{\text {max_qs }}$ is smaller than 2.5 and is valid between $0.45 \leq \frac{c}{h_{m}}<0.75$. Figure 7 shows the relation between $p_{h 3} / p_{h 1}$, $p_{h 1} / p_{v 1}$, and $p_{h 2} / p_{v 1}$ for the case of BW. Similar to the case of SBW, a linear relation is observed between $p_{h 3}$ and $p_{h 1}$. The mean line is shown in Equation 9 (with $\mathrm{s}=0.4772$ ) which is quite different from the line, calculated by Goda's method.

$$
p_{h 3}=0.2 p_{h 1}+1.44
$$

Figure $7 \mathrm{~b}$ shows the relation between $p_{h 1}$ and $p_{v 1}$. In this case, the measured quasi-static pressure at $p_{h 1}$ is higher than the magnitude from the previous case. Here waves are breaking early and approaching the vertical part as a mixture of a water-air jet. Therefore, BW creates rather high or even dynamic pressures on the wall. However, the magnitude of $p_{v 1}$ is lower than the measurements in SBW which is due to the damping effect of air content. The following relation (Equation 10) is proposed between $p_{h 1}$ and $p_{v 1}$ and the logarithm in Equation 11 represents the relation between $p_{h 2}$ and $p_{v 1}$ with and $\mathrm{s}=0.2889$.

$$
\begin{array}{lcl}
p_{v 1} \leq 8 & p_{h 1}=0.24 p_{v 1}+4.7 & \text { with } s=1.8316 \\
p_{v 1}>8 & p_{h 1}=6.5 & \text { with } s=1.7176 \\
\ln \left(p_{h 2}\right)=0.48 \ln \left(p_{v 1}\right)+0.92 &
\end{array}
$$

\section{PRESSURE PROFILES AT BWSAT}

Figure 8 shows the relation of ratios $p_{h 3} / p_{h 1}, p_{h 1} / p_{v 1}$, and $p_{h 2} / p_{v 1}$ for BWSAT. The boundary region is the area between Equation 2 and Equation 3. In this zone, $p_{\max -d y} / p_{\max -q s} \geq 2.5$ and these equations are valid for $0.45 \leq \frac{c}{h_{m}}<0.60$.

In this case, waves collide on the vertical part with a more or less parallel face and only a little amount of air is enclosed. Due to the impact effects, the wave crest breaks up into small droplets and the enclosed air compresses and bursts upwards. The breaking wave creates high dynamic impacts both on the vertical and horizontal parts. The relation between $p_{h 3}$ and $p_{h 1}$ is shown in Figure 8a and the results are compared with the theoretical line derived from the method in Oumeraci et al., 2001 (PROVERBS method). The theoretical line represents the best fit line in the low $p_{h 1}$ region, and it overestimates $p_{h 3}$ values in the high $p_{h 1}$ region. Equation 12 represents the mean of the present data with $\mathrm{s}=0.2614$.

$$
\ln \left(p_{h 3}\right)=0.56 \ln \left(p_{h 1}\right)+0.21
$$

Figure $8 \mathrm{~b}$ represents the relation between $p_{h 1}$ and $p_{v 1}$ which is an inversely proportional relation. Normally, in BWSAT condition, high dynamic pressures are expected both on the vertical and horizontal part for the same wave. However, when a single wave perfectly breaks on the vertical part, it only creates a high impact pressure on the vertical part. Because it loses most of its energy on the vertical part and breaks in to small droplets, it results in relatively low pressures on the horizontal part. Equation 13 shows upper envelope function at $99.6 \%$ of non-exceedance level between $p_{h 1}$ and $p_{v 1}$.

$$
\left(p_{h 1}\right)_{99.6 \%}=1217\left(p_{h 1}\right)^{-0.88}
$$


Figure $8 \mathrm{c}$ shows the relation between $p_{h 2}$ and $p_{v 1}$. As described earlier, the pressure at the location of $p_{h 2}$ is an indirect effect of the impact occurring on the horizontal part. Hence, the latter follows a trend parallel with the increase of $p_{v 1}$. However, it only increases up to a certain value. Beyond this value water cannot compress because one side is open and the water deflects through the open side. Equation 14 represents the formula of the mean line with $\mathrm{s}=0.268$.

$$
\ln \left(p_{h 2}\right)=0.23 \ln \left(p_{h 1}\right)+1.4
$$

Data related to the BWLAT case required more analyses. Therefore, in this chapter we are not suggesting any formula for this particular case.

\section{CONCLUSION}

The pressure distribution due to the violent water wave impacts on a vertical wall with an overhanging horizontal cantilever slab is analyzed based on the results of breaking waves. Both the location and the magnitude of $p_{\max }$ on a vertical structure with an overhanging horizontal cantilever slab are determined. On the vertical part, the non-dimensional term $\left(z_{\max } / h_{s}\right)$ for the location of $p_{\max }$ is gradually decreasing from a point above the SWL to a point below the SWL with the increase of $h_{s}$.

Proper determination of the boundary conditions for the region of dynamic pressures is quite important to develop a reasonable design method. The boundary expressions for the interval of normalized wave height, $\frac{H}{h_{s}}$, which creates a high dynamic impact on the vertical part with the variation of the clearance, $\frac{c}{a}$, are expressed by Equation 2 and Equation 3. This corresponds to the wave range for the cases of BWSAT and BWLAT where $0.67<H / h_{s}<1.19$. In addition, boundaries for the region of dynamic pressure occurring on the horizontal part are shown by Equation 4 and Equation 5.

The spatial distribution of local $p_{\max }$ both on the vertical and horizontal part for cases SBW, BWSAT and BW are analyzed. For the pressure profiles, local $p_{\max }$ at SWL and at the top and bottom of the vertical part $\left(p_{h 1}, p_{h 2}\right.$ and $\left.p_{h 3}\right)$ and at the attached corner of the horizontal part $\left(p_{v 1}\right)$ are considered. For each case, the relation for $p_{h 3} / p_{h 1}, p_{h 1} / p_{v 1}$, and $p_{h 2} / p_{v 1}$ are determined.

In SBW, a linear relation is observed between $p_{h 3}$ and $p_{h 1}$ which complies with the theoretical expression of Goda (1974). In addition, the relation between $p_{h 1}$ and $p_{v 1}$ is also linear for very small values of $p_{v 1}$ and it is constant for higher values of $p_{v 1}$. Furthermore, a logarithmic relation is fitted for the upper envelope line between for $p_{v 1}$ and $p_{h 2}$.

In BW conditions, the relation similar to the case of SBW is observed between $p_{h 3} / p_{h 1}, p_{h 1} / p_{v 1}$, and $p_{h 2} / p_{v 1}$. The measured quasi-static pressure $p_{h 1}$ is higher than the measurements in the previous case. However, the pressure magnitude of $p_{v 1}$ is lower than the measurements in SBW which is due to the damping effect of air content.

The boundary region of BWSAT lies between Equation 2 and Equation 3 and dynamic pressures are measured in this case. A logarithmic relation is proposed between $p_{h 3}$ and $p_{h 1}$ and it is compared with the theoretical line derived from the method in PROVERBS. The theoretical line represents the best fit line in the low $p_{h 1}$ region, and it over estimates $p_{h 3}$ values in the high $p_{h 1}$ region. In addition, an inverse relation is observed between $p_{h 1}$ and $p_{v 1}$. Finally, the relation between $p_{h 2}$ and $p_{v 1}$ is studied and a logarithmic relation is proposed.

\section{REFERENCES}

Goda, Y., 1974, "New wave pressure formulae for composite breakwater", Proc. of 14th Int. Conf. Coastal Eng., Copenhagen, Denmark, ASCE, New York (1974, pp. 1702-1720

Hattori, M.; Arami, A.; Yui, T., 1994, "Wave impact pressure on vertical walls under breaking waves of various type”, Coastal Engineering, v 22, n 1-2, p 79-114, Jan 1994

Hull, P.; Müller, G., 2002, "An investigation of breaker heights, shapes and pressures" Ocean Engineering, v 29, n 1, p 59-79, September 21, 2002

Kirkgoz, M.S., 1982, “Shock pressure of breaking waves on vertical walls” Journal of the Waterway, Port, Coastal and Ocean Division, v 108, $n$ WW1, p 81-95, Feb 1982

Kisacik, D.; Troch, P.; Van Bogaert, P., 2012, "Description of loading conditions due to violent wave impacts on a vertical structure with an overhanging horizontal cantilever slab", Accepted to journal of "Coastal engineering". 
Kortenhaus, A.: and Hocine Oumeraci, H., 1998, "Classification of wave loading on monolithic coastal structures”, Proc. 26th. Int. Coast. Engrg. Conf., ASCE, pp: 867-880 p Year.1998

Oumeraci, H; Kortenhaus, A; Allsop, W; de Groot, M; Crouch, R; Vrijling, H; Voortman, H, 2001, "Probabilistic Design Tools for Vertical Breakwaters", Balkema Publishers, New York.

Partenscky, H., 1988, "Dynamic forces due to waves breaking at vertical coastal structures" Twenty First Costal Eng Conf, p 2504-2518, 1988, Twenty First Costal Eng Conf 